J. Appl. Numer. Optim. 1 (2019), No. 2, pp. 157-165

Available online at http://jano.biemdas.com

https://doi.org/10.23952/jano.1.2019.2.06

\title{
THREE CONVERGENCE RESULTS FOR INEXACT ORBITS OF NONEXPANSIVE MAPPINGS
}

\author{
ALEXANDER J. ZASLAVSKI
}

Department of Mathematics, The Technion - Israel Institute of Technology, 32000 Haifa, Isreal

\begin{abstract}
In this paper we study the convergence of inexact iterates of nonexpansive mappings which take a nonempty, closed subset of a complete metric space into the space, under the presence of summable errors, and generalize the known results in the literature for nonexpansive self-mappings of the complete metric space.
\end{abstract}

Keywords. Banach space; Complete metric space; Inexact iteration; Nonexpansive mapping.

2010 Mathematics Subject Classification. 47H09, 47H10, 54E50.

\section{INTRODUCTION}

In the past fifty-five years, there has been a lot of activities regarding the fixed point theory of nonexpansive (that is, 1Lipschitz) mappings; see, for example, [3, 5, 11, 13, 14, 17, 18, 19, 21, 22, 23, 24, 27, 28] and the references cited therein. These activities stem from Banach's classical theorem [1] concerning the existence of a unique fixed point for a strict contraction. It also covers the convergence of (inexact) iterates of a nonexpansive mapping to one of its fixed points. Since that seminal result, many developments have taken place in this field including, in particular, studies of feasibility and common fixed point problems, which find important applications in engineering and medical sciences [8, 12, 15, 25, 26, 27, 28].

In [5] it was shown that if any exact orbit of a nonexpansive mapping converges to its fixed point, then this convergence property also holds for its inexact orbits with summable errors. This result was obtained for a self-mapping of a complete metric space $X$. In the present paper, we generalize this results for nonexpansive mappings which take a nonempty, closed subset of the complete metric space $X$ into $X$.

\section{Preliminaries}

Let $(X, \rho)$ be a complete metric space. For each $x \in X$ and each nonempty set $B \subset X$, put

$$
\rho(x, B)=\inf \{\rho(x, y): y \in B\} .
$$

For each $x \in X$ and each $r>0$, set

$$
B(x, r)=\{y \in X: \rho(x, y) \leq r\} .
$$

For each mapping $A: X \rightarrow X$, let $A^{0} x=x$ for all $x \in X$.

In [5] it was studied the influence of errors on the convergence of orbits of nonexpansive mappings in metric spaces and it was obtained the following result (see also Theorem 2.72 of [24]).

Theorem 2.1. Let $A: X \rightarrow X$ satisfy $\rho(A x, A y) \leq \rho(x, y)$, for all $x, y \in X$. Let $F(A)$ be the set of all fixed points of $A$ and let, for each $x \in X,\left\{A^{n} x\right\}_{n=1}^{\infty}$ be a sequence converging in $(X, \rho)$. Assume that $\left\{x_{n}\right\}_{n=0}^{\infty} \subset X,\left\{r_{n}\right\}_{n=0}^{\infty} \subset(0, \infty)$ satisfies $\sum_{n=0}^{\infty} r_{n}<\infty$ and

$$
\rho\left(x_{n+1}, A x_{n}\right) \leq r_{n}, n=0,1, \ldots
$$

E-mail address: ajzasl@technion.ac.il.

Received May 24, 2019; Accepted July 30, 2019. 
Then the sequence $\left\{x_{n}\right\}_{n=1}^{\infty}$ converges to a fixed point of $A$ in $(X, \rho)$.

In this paper, we generalize this result for nonexpansive mappings which take a nonempty, closed subset of the complete metric space $X$ into $X$. More precisely, in Section 4, we prove the following result.

Theorem 2.2. Let $K$ be a nonempty closed subset of $X$. Let $A: K \rightarrow X$ satisfy

$$
\rho(A x, A y) \leq \rho(x, y) \text { for all } x, y \in K,
$$

and let $F(A)$ be the set of all fixed points of $A$. For each $x \in X$, if $\left\{A^{n} x\right\}_{n=1}^{\infty}$ is well-defined, then it converges in $(X, \rho)$. Let

$$
\left\{x_{n}\right\}_{n=0}^{\infty} \subset K,
$$

$\tilde{r}>0$, and $\left\{r_{n}\right\}_{n=0}^{\infty} \subset(0, \infty)$ satisfy

$$
\begin{gathered}
\sum_{n=0}^{\infty} r_{n}<\infty, \\
\rho\left(x_{n+1}, A x_{n}\right) \leq r_{n}, n=0,1, \ldots
\end{gathered}
$$

Let

$$
B\left(x_{n}, \tilde{r}\right) \subset K \text { for all sufficiently large natural numbers } n \text {. }
$$

Then the sequence $\left\{x_{n}\right\}_{n=1}^{\infty}$ converges to a fixed point of $A$ in $(X, \rho)$.

Theorem 2.1 finds interesting applications and is an important ingredient in superiorization and perturbation resilience of algorithms; see $[2,4,6,7,9,10,16,20]$ and the references mentioned therein. The superiorization methodology works by taking an iterative algorithm, investigating its perturbation resilience, and then using proactively such perturbations in order to "force" the perturbed algorithm to do in addition to its original task something useful. This methodology can be explained by the following result on convergence of inexact iterates.

Assume that $(X,\|\cdot\|)$ is a Banach space, $\rho(x, y)=\|x-y\|$ for all $x, y \in X$, for each $x \in X$, the sequence $\left\{A^{n} x\right\}_{n=1}^{\infty}$ converges in the norm topology. Assume that $x_{0} \in X,\left\{\beta_{k}\right\}_{k=0}^{\infty}$ is a sequence of positive numbers satisfying

$$
\sum_{k=0}^{\infty} \beta_{k}<\infty,
$$

$\left\{v_{k}\right\}_{k=0}^{\infty} \subset X$ is a norm bounded sequence and, for any integer $k \geq 0$,

$$
x_{k+1}=A\left(x_{k}+\beta_{k} v_{k}\right) \text {. }
$$

Then it follows from Theorem 2.1 that the sequence $\left\{x_{k}\right\}_{k=0}^{\infty}$ converges in the norm topology of $X$ and its limit is a fixed point of $A$. In this case, mapping $A$ is called the bounded perturbations resilient (see [6] and Definition 10 of [9]). In other words, if exact iterates of a nonexpansive mapping converge, then its inexact iterates with bounded summable perturbations converge too.

Now assume that $x_{0} \in X$ and the sequence $\left\{\beta_{k}\right\}_{k=0}^{\infty}$ satisfying (2.6) are given and we need to find an approximate fixed point of $A$. In order to meet this goal, we construct a sequence $\left\{x_{k}\right\}_{k=1}^{\infty}$ defined by (2.7). Under an appropriate choice of the bounded sequence $\left\{v_{k}\right\}_{k=0}^{\infty}$, the sequence $\left\{x_{k}\right\}_{k=1}^{\infty}$ possesses some useful property. For example, the sequence $\left\{f\left(x_{k}\right)\right\}_{k=1}^{\infty}$ can be decreasing, where $f$ is a given function.

\section{Proof of Theorem 2.2}

Set $A^{0} x=x$ for all $x \in K$. It is sufficient to show that the sequence $\left\{x_{n}\right\}_{n=0}^{\infty}$ converges in $(X, \rho)$.

Let $\varepsilon>0$. Since the metric space $(X, \rho)$ is complete, it is sufficient to show that there exists a natural number $k$ such that, for each pair of integers $i, j \geq k$,

$$
\rho\left(x_{i}, x_{j}\right) \leq \varepsilon
$$

We may assume that

$$
\varepsilon \in(0, \tilde{r} / 4)
$$

In view of (2.5), there exists a natural number $k_{1}$ such that

$$
B\left(x_{n}, \tilde{r}\right) \subset K \text { for all natural numbers } n \geq k_{1} .
$$


By (2.3), there exists an integer $k_{0}>k_{1}$ such that

$$
\sum_{i=k_{0}}^{\infty} r_{i}<\varepsilon / 4
$$

By induction, we show that

$$
A^{n} x_{k_{0}} \in K
$$

is well defined for all natural numbers $n$ and that

$$
\rho\left(A^{n} x_{k_{0}}, x_{n+k_{0}}\right)<\varepsilon .
$$

for all integers $n \geq 0$. Assume that $n \geq 0$ is an integer,

$$
A^{i} x_{k_{0}} \in K, i=0, \ldots, n
$$

are well defined and that

$$
\rho\left(A^{n} x_{k_{0}}, x_{n+k_{0}}\right) \leq \sum_{i=k_{0}}^{n+k_{0}} r_{i}-r_{n+k_{0}} .
$$

(It is clear that our assumption holds for $n=0$ ). In view of (3.2) and the inequality $k_{0}>k_{1}$, we have

$$
B\left(x_{n+k_{0}}, \tilde{r}\right) \subset K .
$$

By (3.4), we have

$$
A^{n} x_{k_{0}} \in K
$$

and $A^{n+1} x_{k_{0}} \in X$ is well defined. It follows from (2.1), (2.2), (2.4), (3.5) and (3.6) that

$$
\begin{aligned}
\rho\left(A^{n+1} x_{k_{0}}, x_{n+1+k_{0}}\right) & \leq \rho\left(A^{n+1} x_{k_{0}}, A x_{n+k_{0}}\right)+\rho\left(A x_{n+k_{0}}, x_{n+k_{0}+1}\right) \\
& \leq \rho\left(A^{n} x_{k_{0}}, x_{n+k_{0}}\right)+r_{n+k_{0}} \\
& \leq \sum_{i=k_{0}}^{n+k_{0}} r_{i} \\
& =\sum_{i=k_{0}}^{n+k_{0}+1} r_{i}-r_{n+k_{0}+1} .
\end{aligned}
$$

It follows from (3.1), (3.2), (3.3) and (3.7) that

$$
\begin{gathered}
\rho\left(A^{n+1} x_{k_{0}}, x_{n+1+k_{0}}\right)<\varepsilon<\tilde{r} / 4, \\
A^{n+1} x_{k_{0}} \in K
\end{gathered}
$$

and the assumption made for $n$ also holds for $n+1$. Therefore by induction, we show that

$$
A^{i} x_{k_{0}} \in K \text { for all integers } i \geq 0
$$

and that (3.5) holds for all integers $n \geq 0$. By our assumptions, there exists

$$
z \in F(T)
$$

such that

$$
z=\lim _{n \rightarrow \infty} A^{n} x_{k_{0}} .
$$

It follows from (3.3) and (3.5) that, for all integers $n \geq 0$,

$$
\rho\left(A^{n} x_{k_{0}}, x_{n+k_{0}}\right) \leq \sum_{i=k_{0}}^{n+k_{0}} r_{i} \leq \sum_{i=k_{0}}^{\infty} r_{i}<\varepsilon / 4 .
$$

In view of (3.8), there exists a natural number $n_{1}$ such that, for each integer $n \geq n_{1}$,

$$
\rho\left(A^{n} x_{k_{0}}, z\right) \leq \varepsilon / 4 .
$$

By the relation above and (3.9), we have, for each integer $n \geq n_{1}$,

$$
\rho\left(z, x_{n+k_{0}}\right) \leq \rho\left(z, A^{n} x_{k_{0}}\right)+\rho\left(A^{n} x_{k_{0}}, x_{n+k_{0}}\right) \leq \varepsilon / 4+\varepsilon / 4 .
$$

Thus, for all integers $n, m \geq n_{1}$,

$$
\rho\left(x_{n+k_{0}}, x_{m+k_{0}}\right) \leq \rho\left(x_{n+k_{0}}, z\right)+\rho\left(z, x_{m+k_{0}}\right) \leq \varepsilon / 2 .
$$


This completes the proof of Theorem 2.2.

\section{WEAK CONVERGENCE OF ORBITS}

Let $X$ be a nonempty closed subset of a Banach space $(E,\|\cdot\|)$ with a dual space $\left(E^{*},\|\cdot\|_{*}\right)$ and let $A: X \rightarrow X$ satisfy

$$
\|A x-A y\| \leq\|x-y\| \text { for each } x, y \in X .
$$

As usual, we denote by $A^{0}$ the identity self-mapping of $X$. Consider the following assumptions.

(A1) For each $x \in X$, the sequence $\left\{A^{n} x\right\}_{n=1}^{\infty}$ converges weakly in $X$.

(A2) For each $x \in X$, the sequence $\left\{A^{n} x\right\}_{n=1}^{\infty}$ converges weakly in $X$ to a fixed point of $A$.

The following result was obtained in [5] (see also Theorem 2.73 of [24]).

Theorem 4.1. Assume that (A1) holds. Let $x_{0} \in X$,

$$
\begin{gathered}
\left\{r_{n}\right\}_{n=0}^{\infty} \subset(0, \infty), \sum_{n=0}^{\infty} r_{n}<\infty, \\
\left\{x_{n}\right\}_{n=0}^{\infty} \subset X,\left\|x_{n+1}-A x_{n}\right\| \leq r_{n}, n=0,1, \ldots
\end{gathered}
$$

Then the sequence $\left\{x_{n}\right\}_{n=1}^{\infty}$ converges weakly in X. Moreover, if (A2) holds, then its limit is a fixed point of $A$.

In the present paper we generalize this result for nonexpansive mappings which take a nonempty, closed subset of the complete metric space $X$ into $X$.

Let $K$ be a nonempty closed subset of $E$ and $A: K \rightarrow E$ satisfy

$$
\|A x-A y\| \leq\|x-y\| \text { for each } x, y \in K .
$$

Let $A^{0} x=x$ for all $x \in K$.

We use following assumptions.

(A3) If $x \in K$ and the sequence $\left\{A^{n} x\right\}_{n=1}^{\infty} \subset K$ is well defined, then it converges weakly in $E$.

(A4) If $x \in K$ and the sequence $\left\{A^{n} x\right\}_{n=1}^{\infty} \subset K$ is well defined, then it converges weakly in $E$ to a fixed point of $A$.

Let

$$
F(A)=\{x \in K: A x=x\}
$$

Clearly, $F(A)$ is a closed subset of $E$.

We prove the following result.

Theorem 4.2. Let (A3) hold, $\left\{x_{n}\right\}_{n=0}^{\infty} \subset K, \tilde{r}>0,\left\{r_{n}\right\}_{n=0}^{\infty} \subset(0, \infty)$ satisfy

$$
\begin{gathered}
\sum_{n=0}^{\infty} r_{n}<\infty, \\
\left\|x_{n+1}-A x_{n}\right\| \leq r_{n}, n=0,1, \ldots
\end{gathered}
$$

and

$$
B\left(x_{n}, \tilde{r}\right) \subset K \text { for all sufficiently large natural numbers } n \text {. }
$$

Then the sequence $\left\{x_{n}\right\}_{n=1}^{\infty}$ converges weakly in $E$ and if $K$ is weakly closed, then the limit belongs to $K$. Moreover, if (A4) holds, then the limit is a fixed point of $A$.

Proof. In view of (4.3), there exists a natural number $n_{0}$ such that

$$
B\left(x_{n}, \tilde{r}\right) \subset K \text { for all natural numbers } n \geq n_{0} .
$$

By (4.1), there exists an integer

$$
n_{1}>n_{0}
$$

such that

$$
\sum_{i=n_{1}}^{\infty} r_{i}<\tilde{r} / 4
$$

Let

$$
p \geq n_{1}
$$


be an integer. By induction, we show that $A^{i} x_{p} \in K$ is well defined for all integers $i \geq 0$. Assume that $n \geq 0$ is an integer,

$$
A^{i} x_{p} \in K, i=0, \ldots, n
$$

are well defined and that

$$
\left\|A^{n} x_{p}-x_{n+p}\right\| \leq \sum_{i=p}^{n+p} r_{i}-r_{n+p} .
$$

(It is clear that our assumption holds for $n=0$ ). In view of (4.8), we find that $A^{n+1} x_{p} \in E$ is well defined. It follows from (4.4), (4.5) and (4.7) that

$$
B\left(x_{n+p+1}, \tilde{r}\right) \subset K .
$$

By (4.2), (4.9) and (4.10), we have

$$
\begin{aligned}
\left\|A^{n+1} x_{p}-x_{n+1+p}\right\| & \leq\left\|A^{n+1} x_{p}-A x_{n+p}\right\|+\left\|A x_{n+p}-x_{n+p+1}\right\| \\
& \leq\left\|A^{n} x_{p}-x_{n+p}\right\|+r_{n+p} \\
& \leq \sum_{i=p}^{n+p} r_{i} .
\end{aligned}
$$

It follows from (4.6), (4.7) and (4.11) that

$$
\left\|A^{n+1} x_{p}-x_{n+1+p}\right\|<\tilde{r} / 4 .
$$

In view of (4.4), (4.5), (4.7) and (4.12), we have

$$
A^{n+1} x_{p} \in K .
$$

By (4.11) and (4.13), we have that the assumption made for $n$ also holds for $n+1$. Therefore by induction, we obtain that (4.8) and (4.9) hold for all integers $n \geq 0$. In view of (A3), the sequence $\left\{A^{n} x_{p}\right\}_{n=0}^{\infty}$ converges weakly to $y_{p} \in E$. If the set $K$ is weakly closed, then

$$
y_{p} \in K
$$

and if (A4) holds, then

$$
A y_{p}=y_{p} .
$$

Fix an integer $q \geq 1$. By (4.9), we have

$$
\left\|A^{q} x_{p}-x_{q+p}\right\| \leq \sum_{j=p}^{\infty} r_{j}
$$

It follows that, for each integer $i \geq 0$,

$$
\left\|A^{q+i} x_{p}-A^{i} x_{q+p}\right\| \leq\left\|A^{q} x_{p}-x_{q+p}\right\| \leq \sum_{j=p}^{\infty} r_{j} .
$$

The equations

$$
y_{p}=\lim _{n \rightarrow \infty} A^{n} x_{p} \text { in the weak topology, }
$$

and

$$
y_{q+p}=\lim _{n \rightarrow \infty} A^{n} x_{q+p} \text { in the weak topology }
$$

hold. By (4.17), (4.18) and ((4.19)), for each $f \in E^{*}$ satisfying $\|f\|_{*} \leq 1$, we have

$$
\begin{aligned}
\left|f\left(y_{q}\right)-f\left(y_{q+p}\right)\right| & =\lim _{n \rightarrow \infty}\left|f\left(A^{n+q} x_{p}\right)-f\left(A^{n} x_{q+p}\right)\right| \\
& \leq \limsup _{n \rightarrow \infty}\|f\|\left\|A^{n+q} x_{p}-A^{n} x_{q+p}\right\| \\
& \leq \sum_{j=p}^{\infty} r_{j} .
\end{aligned}
$$

This implies that

$$
\left\|y_{p}-y_{p+q}\right\| \leq \sum_{j=k}^{\infty} r_{j} .
$$

Since the above inequality holds for each integer $p \geq n_{1}$ and each integer $q \geq 1$ and $\sum_{j=0}^{\infty} r_{j}<\infty$, we conclude that $\left\{y_{k}\right\}_{k=1}^{\infty}$ is a Cauchy sequence and there exists

$$
y_{*}=\lim _{k \rightarrow \infty} y_{k}
$$


in the norm topology of $E$. Clearly, if $K$ is weakly closed, then $y_{*} \in K$ and if (A4) holds, then $A y_{*}=y_{*}$. By (4.20) and (4.21), we have

$$
\left\|y_{p}-y_{*}\right\| \leq \sum_{j=p}^{\infty} r_{j}
$$

for all integers $p \geq n_{1}$. In order to complete the proof, it is sufficient to show that $\lim _{k \rightarrow \infty} x_{k}=y_{*}$ in the weak topology. Let $f \in E^{*}$ be a continuous linear functional on $E$ such that $\|f\|_{*} \leq 1$ and let $\varepsilon>0$ be given. It is sufficient to show that $\left|f\left(y_{*}-x_{i}\right)\right| \leq \varepsilon$ for all large enough integers $i \geq 0$. By (4.1), there is an integer $p \geq n_{1}$ such that

$$
\sum_{j=p}^{\infty} r_{j}<\varepsilon / 4 .
$$

By (4.16) and (4.22), for each integer $n \geq 1$, we have

$$
\begin{aligned}
\left|f\left(y_{*}-x_{p+n}\right)\right| & \leq\left|f\left(y_{*}-y_{p}\right)\right|+\left|f\left(y_{p}-A^{n} x_{p}\right)\right|+\left|f\left(A^{n} x_{p}-x_{n+p}\right)\right| \\
& \leq\left\|y_{*}-y_{p}\right\|+\left|f\left(y_{p}-A^{n} x_{p}\right)\right|+\left\|A^{n} x_{p}-x_{n+p}\right\| \\
& \leq \sum_{j=p}^{\infty} r_{j}+\left|f\left(y_{p}-A^{n} x_{p}\right)\right|+\sum_{j=p}^{\infty} r_{j} .
\end{aligned}
$$

Since $y_{p}=\lim _{n \rightarrow \infty} A^{n} x_{p}$ in the weak topology of $E$, there exits a natural number $i_{0}$ such that

$$
\left|f\left(y_{p}-A^{n} x_{p}\right)\right|<\varepsilon / 4 \text { for all natural numbers } n \geq i_{0} .
$$

By (4.23) and (4.25), we have, for each integer $n \geq i_{0}$,

$$
\left|f\left(y_{*}-x_{p+n}\right)\right| \leq \varepsilon / 4+\varepsilon / 4+\varepsilon / 4=3 \varepsilon / 4 .
$$

This completes the proof.

\section{AN EXTENSION OF THEOREM 2.2}

Let $K$ be a nonempty closed subset of a complete metric space $(X, \rho)$. Let $F \subset K$ be a nonempty closed set in $(X, \rho)$ and let $A_{i}: K \rightarrow X, i=0,1, \ldots$ satisfy for all integers $i \geq 0$,

$$
\begin{gathered}
\rho\left(A_{i} x, A_{i} y\right) \leq \rho(x, y) \text { for all } x, y \in K, \\
A_{i} x=x \text { for all } x \in F
\end{gathered}
$$

We use the following assumptions.

(A1) If $x \in K, p \geq 0$ is an integer and the sequence $\left\{\prod_{i=p}^{n} A_{i} x\right\}_{n=p}^{\infty} \subset K$ is well defined, then it converges in $(X, \rho)$.

(A2) If $x \in K, p \geq 0$ is an integer and the sequence $\left\{\prod_{i=p}^{n} A_{i} x\right\}_{n=p}^{\infty} \subset K$ is well defined, then it converges in $(X, \rho)$ and

$$
\lim _{n \rightarrow \infty} \prod_{i=p}^{n} A_{i} x \in F .
$$

We prove the following result.

Theorem 5.1. Let $\tilde{r}>0,\left\{r_{n}\right\}_{n=0}^{\infty} \subset(0, \infty)$ satisfy

$$
\begin{gathered}
\sum_{n=0}^{\infty} r_{n}<\infty, \\
\left\{x_{n}\right\}_{n=0}^{\infty} \subset K, \\
\rho\left(x_{n+1}, A_{n} x_{n}\right) \leq r_{n}, n=0,1, \ldots
\end{gathered}
$$

and

$$
B\left(x_{n}, \tilde{r}\right) \subset K \text { for all sufficiently large natural numbers } n .
$$

Then the sequence $\left\{x_{n}\right\}_{n=1}^{\infty}$ converges in $(X, \rho)$. Moreover, if (A2) holds, then

$\lim _{n \rightarrow \infty} x_{n} \in F$. 
Proof. In view of (5.6), there exists a natural number $n_{0}$ such that

$$
B\left(x_{n}, \tilde{r}\right) \subset K \text { for all natural numbers } n \geq n_{0} .
$$

Let

$$
\varepsilon \in(0, \tilde{r} / 2)
$$

By (5.3), we have that there exists an integer

$$
n_{1} \geq n_{0}
$$

such that

$$
\sum_{i=n_{1}}^{\infty} r_{i}<\varepsilon / 4
$$

By induction, we show that, for all integer $n \geq n_{1}$,

$$
\prod_{i=n_{1}}^{n} A_{i} x_{n_{1}} \in K
$$

is well defined and

$$
\rho\left(\prod_{i=n_{1}}^{n} A_{i} x_{n_{1}}, x_{n+1}\right) \leq \sum_{i=n_{1}}^{n} r_{i} .
$$

It follows from (5.5), (5.7), (5.8) and (5.10) that

$$
\rho\left(A_{n_{1}} x_{n_{1}}, x_{n_{1}+1}\right) \leq r_{n_{1}}<\tilde{r}
$$

and

$$
A_{n_{1}} x_{n_{1}} \in B\left(x_{n_{1}+1}, \tilde{r}\right) \subset K .
$$

Assume that $n \geq n_{1}$ is an integer, $\prod_{i=n_{1}}^{n} A_{i} x_{n_{1}} \in K$ is well defined and (5.12) holds. (By (5.13) and (5.14), our assumption holds for $n=n_{1}$ ). It follows from (5.1), (5.5) and (5.12) that

$$
\begin{aligned}
\rho\left(\prod_{i=n_{1}}^{n+1} A_{i} x_{n_{1}}, x_{n+2}\right) & \leq \rho\left(\prod_{i=n_{1}}^{n+1} A_{i} x_{n_{1}}, A_{n+1} x_{n+1}\right)+\rho\left(A_{n+1} x_{n+1}, x_{n+2}\right) \\
& \leq \rho\left(\prod_{i=n_{1}}^{n} A_{i} x_{n_{1}}, x_{n+1}\right)+r_{n+1} \leq \sum_{i=n_{1}}^{n+1} r_{i} .
\end{aligned}
$$

By (5.8), (5.10) and (5.15), we have

$$
\rho\left(\prod_{i=n_{1}}^{n+1} A_{i} x_{n_{1}}, x_{n+2}\right) \leq \tilde{r}
$$

In view of (5.7) and (5.16), we have

$$
\prod_{i=n_{1}}^{n+1} A_{i} x_{n_{1}} \in B\left(x_{n+2}, \tilde{r}\right) \subset K
$$

It follows from the inclusion above and (5.15) that the assumption made for $n$ also holds for $n+1$. Therefore by induction, we show that

$$
\prod_{i=n_{1}}^{n} A_{i} x_{n_{1}} \in K \text { for all integers } n \geq n_{1}
$$

and that (5.12) holds for all integers $n \geq n_{1}$. From our assumptions, there exists

$$
y=\lim _{n \rightarrow \infty} \prod_{i=n_{1}}^{n} A_{i} x_{n_{1}}
$$

and if (A2) holds, then $y \in F$. In view of (5.17), there exists an integer $n_{2}>n_{1}$ such that for all integers $n \geq n_{1}$,

$$
\rho\left(y, \prod_{i=n_{1}}^{n} A_{i} x_{n_{1}}\right)<\varepsilon / 4 .
$$

It follows from (5.10), (5.12) and (5.18) that

$$
\rho\left(x_{n+p}, y\right) \leq \rho\left(x_{n+p}, \prod_{i=n_{1}}^{n} A_{i} x_{n_{1}}\right)+\rho\left(\prod_{i=n_{1}}^{n} A_{i} x_{n_{1}}, y\right)<\varepsilon / 2 .
$$

Since $\varepsilon$ is any number satisfying (5.8), we conclude that $\left\{x_{i}\right\}_{i=0}^{\infty}$ is a Cauchy sequence and there exists $\lim _{i \rightarrow \infty} x_{i} \in K$. By (5.19), we have $\rho\left(y, \lim _{i \rightarrow \infty} x_{i}\right) \leq \varepsilon / 2$. If (A2) holds, the inequality above implies that $\rho\left(\lim _{i \rightarrow \infty} x_{i}, F\right)<\varepsilon$. Since $\varepsilon$ is any number satisfying (5.8), we conclude that $\lim _{i \rightarrow \infty} x_{i} \in F$. This completes the proof. 


\section{REFERENCES}

[1] S. Banach, Sur les opérations dans les ensembles abstraits et leur application aux équations intégrales, Fund. Math. 3 (1922), 133-181.

[2] H.H. Bauschke, V.R. Koch, Projection methods: Swiss army knives for solving feasibility and best approximation problems with half-spaces, Contemporary Math. 636 (2015), 1-40.

[3] A. Betiuk-Pilarska, T. Domínguez Benavides, Fixed points for nonexpansive mappings and generalized nonexpansive mappings on Banach lattices, Pure Appl. Func. Anal. 1 (2016), 343-359.

[4] D. Butnariu, R. Davidi, G.T. Herman, I.G. Kazantsev, Stable convergence behavior under summable perturbations of a class of projection methods for convex feasibility and optimization problems, IEEE J. Selected Topics in Signal Processing 1 (2007), 540-547.

[5] D. Butnariu, S. Reich, A.J. Zaslavski, Convergence to fixed points of inexact orbits of Bregman-monotone and of nonexpansive operators in Banach spaces, Proceedings of Fixed Point Theory and its Applications, pp. 11-32, Yokahama Publishers, Mexico, 2006.

[6] Y. Censor, R. Davidi, G.T. Herman, Perturbation resilience and superiorization of iterative algorithms, Inverse Prob. 26 (2010), 1-12.

[7] Y. Censor, R. Davidi, G.T. Herman, R.W. Schulte, L. Tetruashvili, Projected subgradient minimization versus superiorization, J. Optim. Theory Appl. 160 (2014), 730-747.

[8] Y. Censor, M. Zaknoon, Algorithms and convergence results of projection methods for inconsistent feasibility problems: a review, Pure Appl. Func. Anal. 3 (2018), 565-586.

[9] Y. Censor, A.J. Zaslavski, Convergence and perturbation resilience of dynamic string-averaging projection methods, Comput. Optim. Appl. 54 (2013), 65-76.

[10] Y. Censor, A. J. Zaslavski, Strict Fejer monotonicity by superiorization of feasibility-seeking projection methods, J. Optim. Theory Appl. 165 (2015), 172-187.

[11] F.S. de Blasi, J. Myjak, S. Reich, A.J. Zaslavski, Generic existence and approximation of fixed points for nonexpansive set-valued maps, Set-Valued Var. Anal. 17 (2009), 97-112.

[12] A. Gibali, A new split inverse problem and an application to least intensity feasible solutions, Pure Appl. Funct. Anal. 2 (2017), 243-258.

[13] K. Goebel, W. A. Kirk, Topics in metric fixed point theory, Cambridge University Press, Cambridge, 1990.

[14] K. Goebel, S. Reich, Uniform convexity, hyperbolic geometry, and nonexpansive mappings, Marcel Dekker, New York and Basel, 1984.

[15] L.G. Gurin, B.T. Poljak, E.V. Raik, ' Projection methods for finding a common point of convex sets, Z. Vychisl. Mat. i Mat. Fiz. 7 (1967), 1211-1228.

[16] G.T. Herman, R. Davidi, Image reconstruction from a small number of projections, Inverse Probl. 2 (2008), 1-17.

[17] J. Jachymski, Extensions of the Dugundji-Granas and Nadler's theorems on the continuity of fixed points, Pure Appl. Funct. Anal. 2 (2017), 657-666.

[18] W.A. Kirk, Contraction mappings and extensions, in: Handbook of Metric Fixed Point Theory, pp. 1-34, Kluwer, Dordrecht, 2001.

[19] R. Kubota, W. Takahashi, Y. Takeuchi, Extensions of Browder's demiclosedness principle and Reich's lemma and their applications, Pure Appl. Func. Anal. 1 (2016), 63-84.

[20] T. Nikazad, R. Davidi, G.T. Herman, Accelerated perturbation-resilient block-iterative projection methods with application to image reconstruction, Inverse Probl. 28 (2012), 1-19.

[21] S. Reich, A.J. Zaslavski, Well-posedness of fixed point problems, Far East J. Math. Sci. Special Issue Part III (2001), 393-401.

[22] S. Reich, A.J. Zaslavski, Generic aspects of metric fixed point theory, in: Handbook of Metric Fixed Point Theory, pp. 557-575, Kluwer, Dordrecht, 2001.

[23] S. Reich, A.J. Zaslavski, Convergence to attractors under perturbations, Commun. Math. Anal. 10 (2011), 57-63.

[24] S. Reich, A.J. Zaslavski, Genericity in nonlinear analysis, Springer, New York, 2014.

[25] W. Takahashi, The split common fixed point problem and the shrinking projection method for new nonlinear mappings in two Banach spaces, Pure Appl. Funct. Anal. 2 (2017), 685-699. 
[26] W. Takahashi, A general iterative method for split common fixed point problems in Hilbert spaces and applications, Pure Appl. Funct. Anal. 3 (2018), 349-369.

[27] A.J. Zaslavski, Approximate solutions of common fixed point problems, Springer Optimization and Its Applications, Springer, Cham, 2016.

[28] A.J. Zaslavski, Algorithms for solving common fixed point problems, Springer Optimization and Its Applications, Springer, Cham, 2018. 